\title{
The effects of sex, book weight, and grip strength on book-carrying styles
}

\author{
PHILIP J. SPOTTSWOOD and GORDON M. BURGHARDT \\ University of Tennessee, Knoxville, Tennessee 37916
}

\begin{abstract}
The relationship between grip strength, book load, and observed book-carrying mode of male and female college students was studied in a natural setting utilizing 350 subjects. It was found that male and female book-carrying modes were significantly different, but that book weights carried by the two sexes did not differ significantly. Male and female grip strengths were significantly different. A chi square analysis further examined the relationships between the four variables. While the relationships that interact to determine the dimorphism observed in book-carrying styles are complex, this study suggests an approach account for the differences observed.
\end{abstract}

Hanaway (1975) and Hanaway and Burghardt (1976) studied the development of male and female bookcarrying styles in a multivariate research design that also compared two levels of socioeconomic status groups (middle income vs. low income) and two different races (black vs. white). Hanaway identified eight different carrying styles, and tabulated observed frequencies of use across ages, sex, economic status, and race. He found that there were significant sex differences for all age groups and reported that the use of specific bookcarrying positions (male characteristic positions and female characteristic positions) became more fixed or stereotyped with increasing age. Hanaway noted that, for males and white females from families with low income, adult-like carrying positions developed linearly. He reported that the major shift from the use of various positions to the female-characteristic positions occurred at the junior high school level for black females and middle-income white females. Hanaway concluded that body shape, grip strength, and book load could not explain the findings, although he did not measure book loads or grip strength directly. He approximated book weights by looking at the number and size of books carried. He inferred that grip strength was not an important variable by noting marked sex differences in book-carrying styles at early ages when, according to Methany (1941), male grip strengths are only $.9 \mathrm{~kg}$ greater than female grip strengths.

Jenni (Note 1) also observed male and female bookcarrying styles cross-culturally (Costa Rica and Montana) and cross-sectionally for age. She reported that males and females do have characteristic sex-dependent

We gratefully acknowledge the help of the students in the senior author's Child Psychology Laboratory class in assisting with the drawings and data collection. We would also like to thank Joey Conroy for helping with some of the analysis and Tom Hanaway for allowing us to use material gathered in his unpublished dissertation on the development of book-carrying styles. We also thank Mary and Don Jenni for their unpublished data and comments on an earlier draft of this paper. carrying styles that do not develop until after the first grade. Here again no attempt was made to quantify grip strengths or book weights. Haraszti (Note 2) studied the percentage of men and women who carried books "clutched to their chests," and reported that significantly more women used this book-carrying position than men.

This study was initiated as a class project to demonstrate advantages of multivariate, observational experiments in allowing the researcher to study interactions of variables. More importantly, book-carrying is an ubiquitous behavior in many modern cultures and books are perhaps the only item frequently carried by persons of both sexes and all ages.

\section{METHOD}

\section{Development of the Category System}

Pilot observations of book-carrying styles were made by four pairs of undergraduate observers. Their descriptions were compiled, and 11 distinct book-carrying positions were delineated. A careful inspection of the 11 positions, however, revealed that 5 of the carrying positions were either variants of a more common carrying style or were unique carrying styles that had extremely low frequencies of occurrence. The six major styles of bookcarrying are presented in Figure 1, with each figure including both visual and verbal descriptions.

\section{Subjects}

Three hundred and fifty students from the University of Tennessee were subjects in this study. Three samples of 100 students were observed by pairs of undergraduate psychology students in a research methods class. One sample of 50 students was collected by a single undergraduate student. In a separate experiment, five students each collected book-carrying data on 20 women students carrying purses and 20 women students not carrying purses.

\section{Apparatus}

Two household $25 \mathrm{-lb}(11.3 \mathrm{~kg})$ scales were used to weigh the books of each subject included in this study. The scales were recalibrated daily. Two standard $100-\mathrm{lb}(45.4 \mathrm{~kg})$ gripometers were used to test the grip strengths of each subject. 


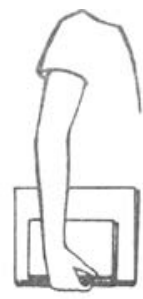

I
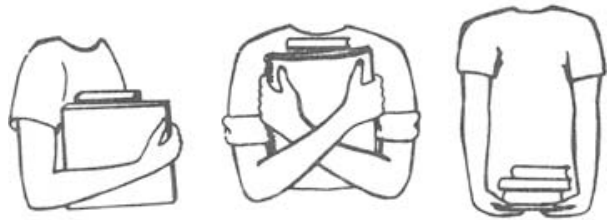

IV
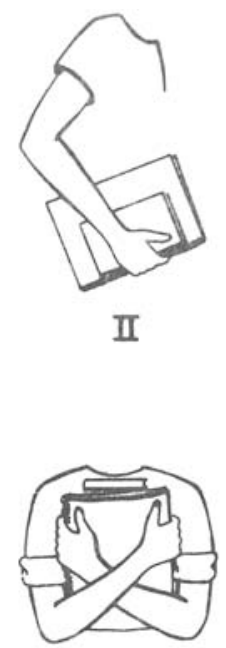

II

立

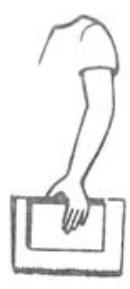

III

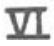

Figure 1. In the three typical male book-carrying positions (1-3), the book weight is mainly supported by the hand grip, with the body used to steady the books. Typical female carrying styles 4 and 5 (70\% of the cases) use the hip-waist area to support the books, with the hand used to steady them.

\section{Procedure}

After learning the verbal definitions of the six book-carrying styles to be used in the study, pairs of observers practiced scoring book-carrying positions. Then the observer teams picked a walkway on the university campus, defined an arbitrary space on that walkway, and observed the book-carrying position and sex of each potential subject who walked across that space. One member of the observation team then approached the subject and asked if he or she would consent to participate in a psychological experiment. If the potential subject replied in the affirmative, he or she was asked to let the observer weigh his or her books. The books were then placed on the experimenters' table, and the subject was asked to squeeze the gripometer as hard as possible with the preferred hand while the other hand was kept hanging freely. The gripometer test was repeated twice and the average score was recorded.

A second procedure was used to sample book-carrying behaviors of females carrying purses, as contrasted with females who did not carry purses. In this situation, the selection of subjects was the same, but no attempt was made to measure grip strength or book weight.

Inter-rater reliabilities for each observer were calculated for the six categories of book-carrying positions. All the observers were shown the same 30 subjects, with each carrying position being presented five times. Each observer's score was compared with a common reliability checker (the first author). The average effective percentage of agreement (see Hartmann, Note 3) for all categories for each observer was: Position $1=95 \%, 2=100 \%$, $3=70 \%, 4=85 \%, 5=80 \%, 6=100 \%$. The average group reliability was $88 \%$. Disagreements, when they occurred, were typically in distinguishing Book-Carrying Position 4 from 5; these positions, at their extremes, graded into each other.

\section{RESULTS AND DISCUSSION}

In this sample, most men used Book-Carrying Position 1 (82\%); most women used Positions $4(51 \%)$ and 5 $(20 \%)$. There was a significant difference between men and women in grip strength (Table 1). The difference in average book-carrying loads for men and women was not significantly different.

A 6 by 2 by 2 by 2 chi-square table was computed to determine possible significant interactions of the four variables. Although the main effects are included in Table 2, they were computed using expected values that were arbitrarily selected. A cutoff point of $17.2 \mathrm{~kg}$ $(38 \mathrm{lbs})$ was selected in dichotomizing the grip strength variable. To differentiate light from heavy book loads, a cutoff point of $910 \mathrm{~g}(2 \mathrm{lbs})$ was established. The six two-way interactions, four three-way interactions, and one four-way interaction were calculated using expected values that were derived from the observed frequencies (see Winer, 1962, pp. 629-632.).

All the main effects were significant at $\alpha=.001$. We conclude that the frequency of students' book-carrying positions is not equally distributed. Given the arbitrary selection of a cutoff point of $17.2 \mathrm{~kg}$ for grip strength, we further conclude that the total sample of 350 students was unevenly distributed around the cutoff point. The weight of books carried by the students also

Table 1

Book Weight and Grip Strength as a Function of Sex

\begin{tabular}{|c|c|c|c|c|}
\hline & & Male & Female & \\
\hline A. Book Weight (kg) & $\begin{array}{l}\text { Mean } \\
\text { SD }\end{array}$ & $\begin{array}{l}1.83 \\
1.19\end{array}$ & $\begin{array}{l}1.71 \\
0.94\end{array}$ & $\begin{array}{l}\mathrm{t}=.111 \\
\mathrm{p}=\mathrm{n} . \mathrm{s} .\end{array}$ \\
\hline B. Grip Strength (kg) & $\begin{array}{l}\text { Mean } \\
\text { SD }\end{array}$ & $\begin{array}{r}22.64 \\
3.43\end{array}$ & $\begin{array}{r}13.76 \\
2.13\end{array}$ & $\begin{aligned} \mathrm{t} & =2.971 \\
\mathrm{p} & <.01\end{aligned}$ \\
\hline
\end{tabular}

Table 2

An Analysis of Factors Affecting Book-Carrying Positions

\begin{tabular}{lccc}
\hline \multicolumn{1}{c}{ Source } & $\begin{array}{c}\text { Chi-Square } \\
\text { Value }\end{array}$ & df & $\alpha$ Leve \\
\hline A. Main Effects* & & & \\
A (Grip Strength) & 14.81 & 1 & .001 \\
B (Book Position) & 408.8 & 5 & .001 \\
C (Weight) & 116.6 & 1 & .001 \\
D (Sex) & 15.64 & 1 & .001
\end{tabular}

B. Interactions

\begin{tabular}{lrrrc} 
A by B & 174.34 & 5 & .001 & 22.66 \\
A by C & .52 & 1 & n.s. & .067 \\
A by D & 254.79 & 1 & .001 & 33.12 \\
B by C & 34.59 & 5 & .001 & 4.50 \\
B by D & 217.13 & 5 & .001 & 28.23 \\
C by D & .59 & 1 & n.s. & .077 \\
A by B by C & 28.57 & 5 & .001 & 3.06 \\
A by B by D & 27.3 & 5 & .001 & 3.55 \\
A by C by D & .21 & 1 & n.s. & .027 \\
B by C by D & 17.82 & 5 & .01 & 2.32 \\
A by B by C by D & 18.40 & 5 & .01 & 2.39 \\
Total Chi Square & 769.26 & 47 & & \\
\hline
\end{tabular}

* Main effects included here are calculated using a priori expected values. They are not included in the total chi square based on expected values calculated from the observed frequencies. 
was not evenly distributed around the 910-g cutoff point, with 276 out of 300 students carrying book loads that weighed more than $910 \mathrm{~g}$. Finally, we conclude that the ratio of males to females at the University of Tennessee was not evenly distributed.

The appropriateness of the a priori assumptions of the expected values for testing the main effects are questionable. The interpretation of the interactions, however, where the expected values are calculated from the row, column, block, and superblock totals, is more meaningful. The total chi-square value should be equal to the sum of all the interactions, and in fact this was the case.

The Grip Strength by Book-Carrying interaction (A by B) was significant at $\mathrm{p}=.001$, and accounted for $22.7 \%$ of the total variance using $5 \mathrm{df}$. Clearly, the relationship between these two variables is strong. The Grip Strength by Sex interaction (A by D) should be highly significant, since we know that grip strengths are bimodally distributed, with males having a significantly greater grip strength than females. The chi-square value for this interaction was the largest of all the two-way interactions, was significant at $\mathrm{p}=.001$, and accounted for $33 \%$ of the total variance while using only $1 \mathrm{df}$. The Book-Carrying by Weight interaction (B by $\mathrm{C}$ ) was also significant, with $\mathrm{p}=.001$, although the interaction accounted for only $4.5 \%$ of the total variance and used 5 df. The Book-Carrying Position by Sex interaction (B by $D$ ) was significant at $p=.001$, and the interaction accounted for more than $28 \%$ of the total variance using $5 \mathrm{df}$. The chi-square value for the Book Weight by Sex (C by D) interaction was not significant. Both sexes typically carried similar book loads and a significant interaction had not been expected.

There were four three-way interactions. Only one three-way interaction was not significant, the Grip Strength by Book Weight by Sex interaction (A by C by $D)$. This was the only three-way interaction that did not include the book-carrying position variable. The other three-way interactions (A by B by C, A by B by D, and $B$ by $C$ by $D$ ) were all significant, although they each explained less than $4 \%$ of the total variance while using $15 \mathrm{df}$. It is interesting that the $\mathrm{B}$ by $\mathrm{C}$ by $\mathrm{D}$ interaction, where grip strength was not a factor, was not as highly significant as were the A by $\mathrm{B}$ by $\mathrm{C}$ and $\mathrm{A}$ by $\mathrm{B}$ by $\mathrm{D}$ interactions.

The four-way interaction was also significant. This interaction, however, much like the three-way interactions, accounted for very little of the total variance explained.

A possible confounding variable that might explain differential male-female carrying styles involved the fact that most women carry purses, whereas most men do not. The possibility that differences observed between men and women were, at least in part, due to this factor was also empirically tested. If carrying purses affected female carrying modes, then the distribution of women carrying purses should differ from women not carrying them. A chi-square test of independence was used to determine whether the observed distribution for 100 women with purses differed from the observed distribution of book-carrying positions of 100 women without purses. The computed chi-square was 6.49 , $\mathrm{df}=1$, n.s. We conclude that carrying purses did not significantly alter the way women in this sample carried their books.

The conclusions are as interesting and as complex as we had first imagined. Grip strength and book weight do not adequately explain the observed sex differences in book-carrying styles. Book weights do not differ significantly for males and females. Further, grip strengths are greater than the book weights carried by at least a factor of 10 . How, then, do physical factors interact with social-cultural factors in determining such a striking dimorphism? To suggest any intricate theory is possible, but premature (Hanaway \& Burghardt, 1976). The relationship of female book-carrying styles to infantcarrying styles in various cultures is a topic worthy of exploration.

\section{REFERENCE NOTES}

1. Jenni, M. A. Sex differences in carrying behavior. Unpublished manuscript. University of Montana, 1975.

2. Haraszti. L. Dimorphism in carrying books. Unpublished paper, University of Chicago. 1972.

3. Hartmann. D. P. Considerations in the choice of interobserver reliability estimates. Unpublished preprint. University of Utah. 1974.

\section{REFERENCES}

Goodman, L. A., \& Kruskal, W. H. Measures of association for cross classifications. Journal of the American Statistical Association, 1954, 49, 732-764.

Hanaway, T. P. The development of gendered book-carrying behavior. Unpublished doctoral dissertation, University of Tennessee, 1975.

Hanaway. T. P., \& Burghardt, G. M. The development of sexually dimorphic book-carrying behavior. Bulletin of the

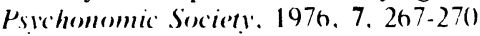

Methany, E. The present status of strength testing for elementary school and preschool age. Research Quarterly, $1941,12,115-130$.

Winer, B. J. Statistical principles in experimental design. New York: McGraw-Hill, 1962.

(Received for publication March 21, 1976.) 